\title{
Towards Quantum Image Processing for Electron Microscopy
}

Roberto dos Reis ${ }^{1}$, Vinayak Dravid ${ }^{1}$ and Stephanie Ribet $^{2}$

${ }^{1}$ Department of Materials Science and Engineering, Northwestern University, United States, ${ }^{2}$ United States

The processing of digital images is continuously gaining in volume and relevance, with associated increasing demands on data storage, transmission, and processing power. Recent advances in transmission electron microscopy instrumentation, especially in detector technology, have propelled data production across a range of modalities. Nowadays, for example, one can imagine generating up to $200 \mathrm{~Tb} / \mathrm{hr}$ by utilizing direct electron detectors [1] requiring smart approaches to extract scientific meaningful information. Despite many progress in microscopy data interpretation with the help of artificial intelligence (AI) and machine learning (ML) methods [1,2], the challenges associated with the growing data volumes for data interpretation remain abundant. This is expected to further exacerbate with a meteoritic rise in in-situ/operando measurements and associated challenges in data mining, analytics, and other computational needs.

Since its genesis in 1982, when Feynman proposed a novel computation model named quantum computers [3], quantum computation has appeared in various areas of computer science including image processing [4]. While still in its infancy, quantum imaging process (QIP) have already presented enormous opportunities to overcome inefficient tasks on classical computers [4], such as efficient pixel storage, retrieval, and transmission. For example, it has been shown that the quantum versions of processing transforms, such as Fourier transforms and discrete cosine transforms, perform more efficiently in quantum computation compared to their classical version [5]. In this work, we will present examples on how to prepare images and implement operations based on quantum states in electron microscopy data.

Quantum computers use qubits, which can be any two-level quantum system in any proportions of both states ( 0 or 1$)$ at once, a concept known as superposition (Figure 1). Today, the available quantum computers represent data using the electronic quantum states of artificial atoms known as superconducting transmon qubits. Various schemes have been proposed to represent images using qubits, and the chosen quantum representation model is key to determine the types of processing tasks and how well they can be performed. Among these, Flexible Representation of Quantum Images (FRQI) [6], novel enhanced quantum representation (NEQR) [7] and Quantum Probability Image Encoding (QPIE) are the most used. (Figure 1B). FRQI captures the essential information about the colors as well as the corresponding positions of every point in an image and integrates them into a quantum state. NEQR uses the basis state of a qubit sequence to store the grayscale value of every pixel, as compared to the angle encoded FRQI qubit. Reducing the number of qubits necessary, QPIE allows for efficient encoding of rectangular images, but faces shortcomings when trying to precisely extract the original image.

An example of application of QIP to detect and match features in electron micrographs is presented in Figure 2. We utilize a typical high resolution scanning transmission electron microscopy (STEM) image obtained from a two-dimensional structure $\mathrm{MoS}_{2} / \mathrm{WS}_{2}$ lateral heterojunction [9]. First, a high resolution annular dark field STEM (ADF-STEM) image (Figure 2A) was reduced from 4096 by 4096 pixels to a 16 by 16 pixels. This reduction is necessary due to limited access to qubits $(<32)$. Then, we quantum encoded the 16 x16 image using the FRQI representation. 
To certify that our encoding was correct, we applied a matching process based on SWAP method[10] using the circuit in Figure 2B. This process is based on computing the overlap between two quantum states, in this case $(\rho)$ and $(\sigma)$. Figure 2 C presents the final quantum matched image to the initial classical $16 \times 16$ reduced image in Figure 2A. The ability to represent a classical image in a quantum framework is the initial step for more complex transformations.

Nowadays, there are various open-source frameworks such as the IBM-Q [11] and Google's Cirq [12] and OpenFermion, [13] where the quantum algorithms can be tested on actual qubits. As the amount of data generated in S/TEM experiments increases greatly, we envision a surge in new methodologies that leverage QIP and these new frameworks to overcome future hurdles in data storage, safe transmission, and processing. The presentation will cover the pros and cons, as well as general considerations for the efficacy of quantum computing in aid of classical electron microscopy data.

This work made use of the EPIC facility of Northwestern University's NUANCE Center, which has received support from the SHyNE Resource (NSF ECCS-2025633), the IIN, and Northwestern's MRSEC program (NSF DMR-1720139). We acknowledge use of the IBM Q for this work. The views expressed are those of the authors and do not reflect the official policy or position of IBM or the IBM Q team.

A

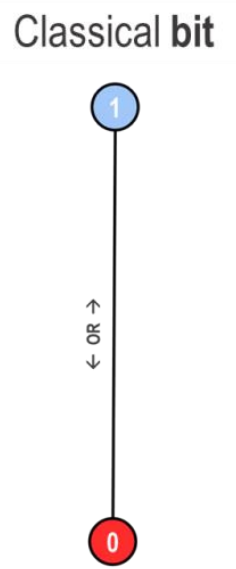

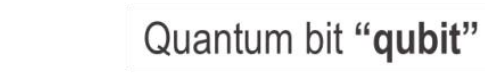

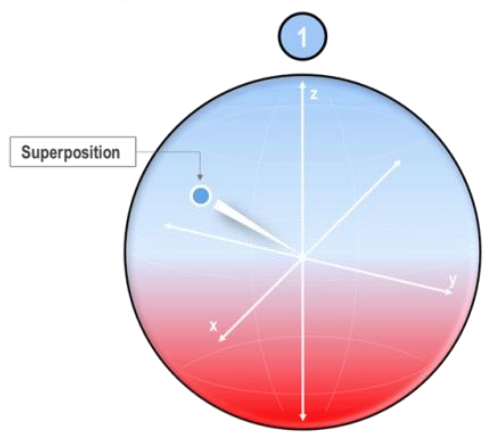

(0)

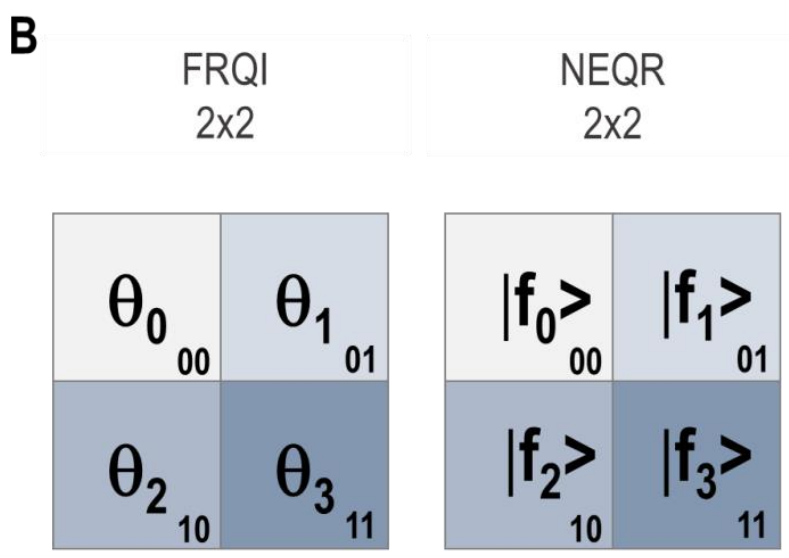

Figure 1. (A) Classical bit versus qubit representation in the Bloch sphere. A qubit can be in a state other than $\mid 0>$ or $\mid 1>$. It is also possible to form linear combinations of states (superpositions). (B) Two different image representations using qubits. A $2 \times 2$ FRQI quantum image and its quantum state, where $\theta$ is the spin's vector angle in the Bloch sphere. The NEQR quantum image uses basis state $\mid \mathrm{fi}>$ of qubit sequence to represent the grayscale of pixels instead of probability amplitude of a single qubit used in the FRQI representation. 

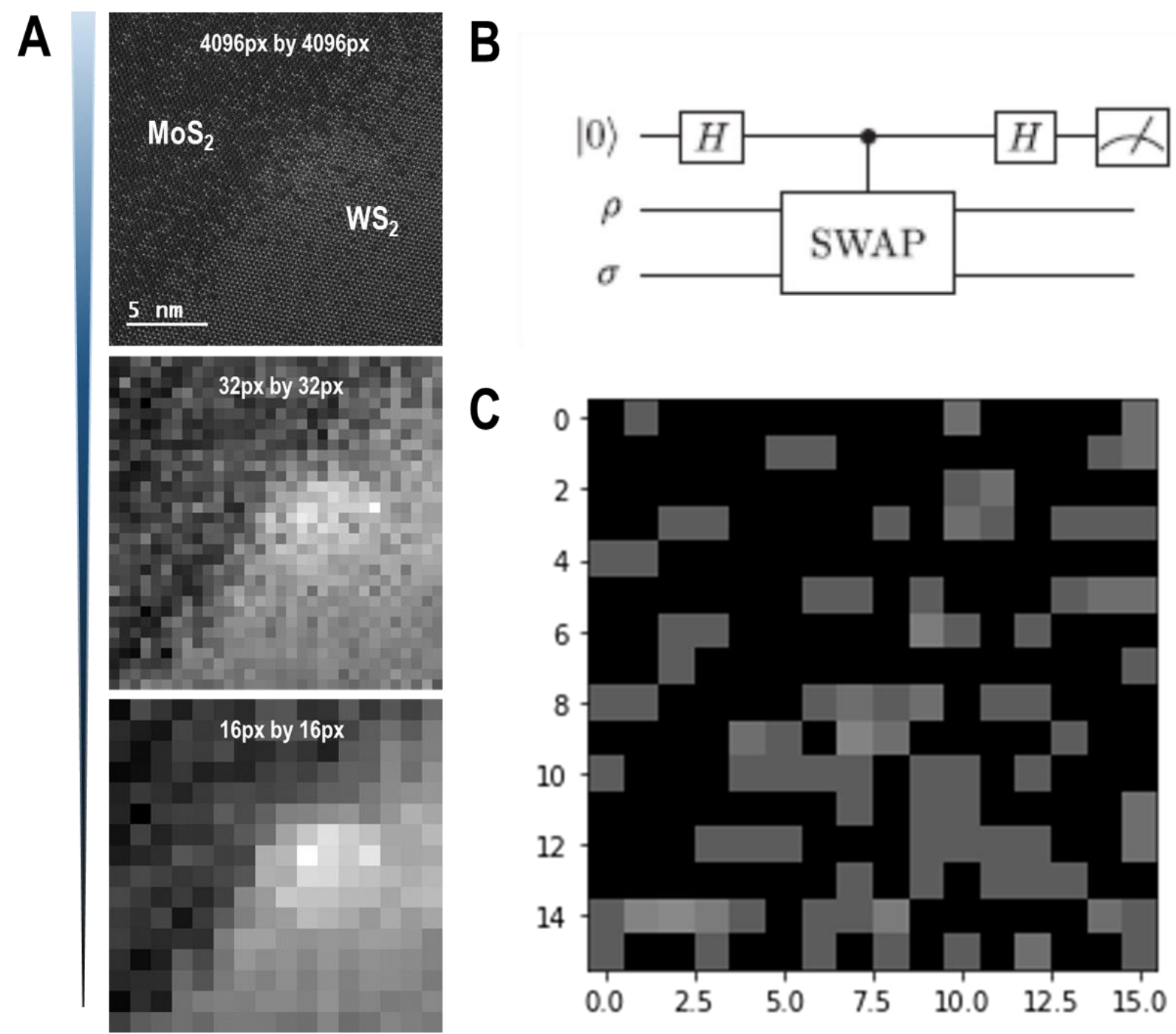

Figure 2. Quantum image representation applied to a STEM-ADF image. (A) Reduction of the collected ADF image to 16x16 pixels. (B) A canonical Swap Test circuit where H indicates a Hadamard gate. (c) Final quantum matched image.

\section{References}

[1] Spurgeon, S. R., et al. (2020). Nature materials, 1-6.

[2] Voyles, P. M. (2017). Current Opinion in Solid State and Materials Science, 21(3), 141-158.

[3] Feynman, R. P. (1982). Int. J. Theor. Phys, 21(6/7).

[4] Vlasov, A. Y. (1997). arXiv preprint quant-ph/9703010.

[5] Nielsen, Michael A., and Isaac Chuang. (2002). Quantum computation and quantum information : 558-559.

[6] Le, P., Dong, F., Hirota, K. (2011). Quantum Inf. Process. 10(1), 63-84.

[7] Zhang, Y., Lu, K., Gao, Y., Wang, M. (2013). Quantum Inf. Process. 12(8), 2833-2860.

[8] Xi-Wei Yao et al. (2017). Phys. Rev. X 7, 031041.

[9] Yasaei, P., et al. (2019). Advanced Materials, 31(24), 1808244.

[10] Cincio, L., Subaşı, Y., Sornborger, A. T., \& Coles, P. J. (2018). New Journal of Physics, 20(11), 113022. 
[11] IBM Quantum. https://quantum-computing.ibm.com/, 2021

[12] Cirq Developers. (2020, October 2). quantumlib/Cirq: Cirq v0.9.1 (Version v0.9.1). Zenodo. http://doi.org/10.5281/zenodo.4064322

[13] OpenFermion: The Electronic Structure Package for Quantum Computers. arXiv:1710.07629. 